\title{
A Permutation Test for Compound Symmetry with Application to Gene Expression Data
}

\author{
Tracy L. Morris \\ University of Central Oklahoma, tmorris2@uco.edu \\ Mark E. Payton \\ Oklahoma State University, mpayton@okstate.edu \\ Stephanie A. Santorico \\ University of Colorado Denver, Stephanie.Santorico@ucdenver.edu
}

Follow this and additional works at: http:// digitalcommons.wayne.edu/jmasm

Part of the Applied Statistics Commons, Social and Behavioral Sciences Commons, and the Statistical Theory Commons

\section{Recommended Citation}

Morris, Tracy L.; Payton, Mark E.; and Santorico, Stephanie A. (2011) "A Permutation Test for Compound Symmetry with Application to Gene Expression Data," Journal of Modern Applied Statistical Methods: Vol. 10 : Iss. 2 , Article 6. DOI: $10.22237 /$ jmasm/1320120300

Available at: http://digitalcommons.wayne.edu/jmasm/vol10/iss2/6 


\section{A Permutation Test for Compound Symmetry with Application to Gene Expression Data}

\author{
Tracy L. Morris \\ University of Central Oklahoma, \\ Edmond, OK
}

\author{
Mark E. Payton \\ Oklahoma State University, \\ Stillwater, OK
}

\author{
Stephanie A. Santorico \\ University of Colorado Denver, \\ Denver, CO
}

The development and application of a permutation test for compound symmetry is described. In a simulation study the permutation test appears to be a level- $\alpha$ test and is robust to non-normality. However, it exhibits poor power, particularly for small samples.

Key words: Compound symmetry, covariance matrix, gene expression, intraclass correlation, microarray, nonparametric inference, permutation test.

\begin{abstract}
Introduction
Determining the underlying covariance or correlation structure of a data set can be challenging. The classical parametric method of testing for some hypothesized covariance structure involves using a likelihood ratio statistic that converges in distribution to a Chisquare random variable (Wilks, 1946). One common covariance structure, in which all of the variances are equal and all of the covariances are equal, is compound symmetry. One of the requirements of the likelihood ratio test (LRT) for compound symmetry is that the data be sampled from a multivariate normal population. Because the LRT is not robust to departures from normality (Huynh \& Mandeville, 1979; Keselman, et al., 1980)a nonparametric test for compound symmetry would be very useful. In particular, permutation tests (PTs) have minimal to no distributional
\end{abstract}

Tracy L. Morris is an Assistant Professor in the Department of Mathematics and Statistics. Email her at: tmorris2@uco.edu. Mark E. Payton is a Professor in the Department of Statistics. Email him at: mark.payton@okstate.edu. Stephanie A. Santorico is an Associate Professor in the Department of Mathematical and Statistical Sciences. Email her at: stephanie.santorico@ucdenver.edu. assumptions, do not require random samples and allow any combination of sample size and number of variables:

Existing Tests for Compound Symmetry

Wilks (1946) was the first to develop a test for compound symmetry. This is a test of $\mathrm{H}_{0}: \boldsymbol{\Sigma}=\boldsymbol{\Sigma}_{C S}$, where

$$
\boldsymbol{\Sigma}_{C S}=\sigma^{2}\left[(1-\rho) \mathbf{I}_{p}+\rho \mathbf{1}_{p} \mathbf{1}_{p}^{\prime}\right]
$$

$\sigma^{2}$ is the common variance; $\rho$ is the common pairwise correlation; $\mathbf{I}_{p}$ is the $p \times p$ identity matrix; and $\mathbf{1}_{p}$ is a $p \times 1$ unit vector. The classical approach to testing for compound symmetry involves the use of a LRT. Let $\mathbf{x}_{i}, i=1, \ldots, n$ be $p$-component vectors distributed according to $\mathrm{N}_{p}(\boldsymbol{\mu}, \boldsymbol{\Sigma})$. The LRT criterion for this test is given by

$$
\lambda=\frac{|\hat{\boldsymbol{\Sigma}}|^{n / 2}}{\left[\left(s^{2}\right)^{p}(1-r)^{p-1}(1+(p-1) r)\right]^{n / 2}},
$$

where $\hat{\boldsymbol{\Sigma}}$ is the maximum likelihood estimator (MLE) of $\boldsymbol{\Sigma}$ under $\mathrm{H}_{\mathrm{a}}: \boldsymbol{\Sigma} \neq \boldsymbol{\Sigma}_{C S}$ and $s$ and $r$ are the MLEs of $\sigma$ and $\rho$, respectively, under $\mathrm{H}_{0}$.

Wilks (1946) determined the exact distribution of $\lambda^{2 / n}$ for $p=2$ and 3 ; however, the derivation of the exact distribution for larger values of $p$ is too complex to be of practical use. 


\section{A PERMUTATION TEST FOR COMPOUND SYMMETRY}

Therefore, the asymptotic distribution is most commonly used. Specifically, $-n \log \lambda^{2 / n}$ is asymptotically distributed as a Chi-square random variable with $\frac{1}{2} p(p+1)-2$ degrees of freedom. As with other LRTs, this is a good approximation when $n$ is considerably larger than $p$, but is poor when $n$ is close to $p$. Therefore, the corrected LRT (CLRT) derived by Box (1950) is preferred. Box showed that $-(n-1) C \log \lambda^{2 / n}$ is asymptotically distributed as a Chi-square random variable with $\frac{1}{2} p(p+1)-2$ degrees of freedom where

$$
C=1-\frac{p(p+1)^{2}(2 p-3)}{6(n-1)(p-1)\left(p^{2}+p-4\right)}
$$

The LRT for compound symmetry is actually just an extension of an earlier test of $\boldsymbol{\Sigma}=\sigma^{2} \mathbf{I}$ developed by Mauchly (1940). Consequently, the LRT for compound symmetry suffers from the same limitations as Mauchly's test. Specifically, it is not a level- $\alpha$ test (Boik, 1975; Cornell, et al., 1992), is not robust to nonnormality (Huynh \& Mandeville, 1979; Keselman, et al., 1980), and requires $n>p$. The CLRT alleviates the problems with the type I error rate (except when $n$ is close to $p$ ). It is not, however, robust to non-normality, and also requires $n>p$.

Wilks' (1946) work was subsequently extended. Lee, Krishnaiah and Chang (1976) determined that the Chi-square approximation for the distribution of the likelihood ratio statistic for compound symmetry is adequate for so-called practical purposes, and Votaw (1948) developed a test for compound symmetry in subsets of variates. Still other authors have explored similar tests for the structure of correlation rather than covariance matrices (Aitkin, 1969; Aitkin, Nelson \& Reinfurt, 1968).

Tests for compound symmetry based on spatial signs and ranks have been developed more recently. Marden (1999) introduced one such rank-based test utilizing the differences between the estimated variances and covariances under the alternative hypothesis and the estimated variances and covariances under the null hypothesis. Two subsequent studies extended this work. The first used a permutation testing procedure where the usual LRT statistic was computed for the spatial ranks (Gao \& Marden, 2001). In the second, a Hotelling $T^{2}-$ type statistic was derived and shown to converge in distribution to a Chi-square random variable (Marden \& Gao, 2002). The latter article also presents a similar test based on spatial signs. Marden \& Gao performed a small simulation study $(n=100 \& p=3)$ for these tests and found both the rank and sign tests to be level- $\alpha$ tests when simulating data from spherically symmetric distributions.

Other authors have considered tests for sphericity based on spatial signs and ranks (Hallin \& Paindaveine, 2006; Sirkiä, Taskinen, Oja \& Tyler, 2009). These tests can also be used to test for compound symmetry by first applying an appropriate data transformation. All of these rank and sign tests are superior to the LRT for compound symmetry in that they broaden the family of distributions to which a test for compound symmetry can be applied. They are also applicable in cases in which $n \leq p$. Unfortunately, these tests still have distributional assumptions: they require that data be sampled from a multivariate elliptical distribution.

\section{Methodology}

When the assumptions of parametric procedures are violated, PTs have been used as alternatives. Specifically, PTs reduce or eliminate distributional assumptions (Fisher, 1936; Good, 2005) and allow the use of nearly any test statistic; they are also valid for any combination of $n$ and $p$. As with any statistical procedure, however, PTs have limitations. The greatest of which is that they can be computationally intensive even for moderate sample sizes. With continued advances in technology, PTs have become more feasible for larger sample sizes; however, there still exists a limit at which the computing time required to examine all possible permutations of the data is prohibitive. In such cases, a random sample of permutations may be selected to compute an approximate p-value (Dwass, 1957). These tests are commonly known as Monte Carlo PTs (MCPT). 


\section{MORRIS, PAYTON \& SANTORICO}

Given the benefits of PTs and the limitations of LRTs for testing for the structure of a covariance matrix, it is the purpose of this research to develop a PT for compound symmetry. Before describing this test, note that covariance matrices are invariant to changes in location. Therefore, it was assumed throughout this study that the variable means are equal. If the variable means are unequal, the raw data can be easily centered by calculating $\mathbf{x}_{i}-\boldsymbol{\mu}$ or $\mathbf{x}_{i}-\overline{\mathbf{x}}$ depending on whether $\boldsymbol{\mu}$ is assumed known or unknown, respectively.

\section{Proposed PT Test for Compound Symmetry}

Let $\quad \mathbf{x}_{i}, \quad i=1, \ldots, n \quad$ be identically distributed, $p$-variate vectors of observations on each of $n$ subjects. The objective is to test $\mathrm{H}_{0}: \boldsymbol{\Sigma}=\boldsymbol{\Sigma}_{C S}$ where $\boldsymbol{\Sigma}$ is the covariance matrix of the distribution of $\mathbf{x}_{i}$, and $\boldsymbol{\Sigma}_{C S}$ has the compound symmetry structure given in (1). Good (2005) argues that the observations within each vector are exchangeable if either (i) the observations are independent, or (ii) they are normally distributed with equal covariances. The first of these conditions is a special case of compound symmetry, called sphericity, in which the variances are all equal and the covariances are all zero. In this case, the PT makes no distributional assumptions. The second set of conditions requires multivariate normality with equal covariances. Under the null hypothesis, the covariances are assumed equal and it appears from the simulation results presented herein that a weaker distributional assumption may be sufficient for practical purposes. Specifically, it appears that equivalent marginal distributions will suffice.

Because covariance matrices are symmetric, one possible test statistic can be computed by summing the absolute differences between the elements on or above the diagonal of the covariance matrix obtained from the observed data and the elements on or above the diagonal of the hypothesized covariance matrix estimated from the observed data. In matrix notation:

$$
D=\mathbf{1}_{\frac{1}{2} p(p+1)}^{\prime}\left|\operatorname{vec}\left(\boldsymbol{\Sigma}_{o b s}-\hat{\boldsymbol{\Sigma}}_{C S}\right)\right|,
$$

where $\boldsymbol{\Sigma}_{o b s}$ is the covariance matrix obtained from the observed data;

$$
\hat{\boldsymbol{\Sigma}}_{C S}=\overline{s^{2}}\left[(1-\bar{r}) \mathbf{I}_{p}+r \mathbf{1}_{p} \mathbf{1}_{p}^{\prime}\right]
$$

$\operatorname{vec}(\mathbf{M})$ is a vector of the elements on or above the diagonal of $\mathbf{M}$; and $\overline{s^{2}}$ and $\bar{r}$ are the means of the sample variances and correlations, respectively. This test statistic is computed for each possible permutation of the data and the proportion of test statistic values greater than or equal to the one obtained from the original data is the p-value. Note that $D$ can also be used to test for a specific common variance and/or correlation by substituting the specified value for $\overline{s^{2}}$ and/or $\bar{r}$, respectively, rather than estimating these values as described previously.

Type I Error

\section{Results}

One-thousand simulations were run using $\mathrm{R}$ version 2.10 .1 (R, 2009) for various combinations of $n(=5,10,25,50,100)$ and $p$ $(=3,5,10,20)$. Due to the extremely large number of permutations required to carry out the PTs for any reasonable values of $n$ and $p$, MCPTs were used in the simulations. For each simulation, a $p$-variate data set was generated and the MCPT, CLRT and sign test for sphericity (SIGN) were performed. The sign test for sphericity is available in the SpatialNP package for $\mathrm{R}$ (Sirkiä, Nordhausen \& Oja, 2009).

One-thousand random permutations of the centered data were sampled for each MCPT. In practice, a much larger sample of permutations would be used for individual tests (usually 10,000 permutations); however, for a simulation study of this size, such a large number proved to be prohibitive. Therefore, 1,000 permutations were sampled for each MCPT based on the suggestions of Jöckel (1986) and Manly (1997). For the CLRT and SIGN test, the asymptotic Chi-square distributions were used to determine approximate $5 \%$ critical values.

Four different multivariate distributions (normal, uniform, double exponential and twoparameter exponential) were investigated. For 


\section{A PERMUTATION TEST FOR COMPOUND SYMMETRY}

the multivariate normal distribution, data were generated in $\mathrm{R}$ using the mvrnorm function within the MASS add-on package (Venables \& Ripley, 2002). For the multivariate uniform distribution data were generated using a procedure described in Falk (1999), and for the multivariate double exponential and twoparameter exponential distributions a procedure described in Vale and Maurelli (1983) was used.

The simulated type I error rates for the tests of compound symmetry are displayed in Figure 1. Simulations were run for $n=5,10,15$, $25,50,100, p=3, \sigma^{2}=9$, and $\rho=0.6$. For normally distributed data, the three tests are comparable with respect to the simulated type I error rates, with the CLRT and SIGN test appearing to be slightly conservative, particularly for small samples. The MCPT appears to be fairly robust to non-normality, especially when the underlying distribution is symmetric (normal, uniform, double exponential); however, in the case of the twoparameter exponential data, the MCPT appears to be too liberal with respect to the simulated type I error rates, especially for small samples. The CLRT appears to be too conservative for uniform data and much too liberal for double exponential and two-parameter exponential data, in the latter case achieving a simulated type I error rate as high as 0.352 for $n=100$.

These results are consistent with those of Huynh and Mandeville (1979) who performed a simulation study of Mauchly's (1940) test of sphericity and found that for light-tailed distributions the LRTs were conservative and for heavy-tailed distributions, the type I error rates exceeded the nominal rate. The SIGN test performs very well with respect to the simulated type I error rates for double exponential data; however, the simulated type I error rates are extremely high for uniform (as high as 1.000 for $n=50$ ) and two-parameter exponential data (as high as 0.604 for $n=100$ ). This is undoubtedly due to the assumption of the SIGN test that the data be sampled from a multivariate elliptical distribution.

One disadvantage of the LRTs is that they do not exist when $p \geq n$; due to this, type I error rates tend to inflate as $p$ approaches $n$. Figure 2 displays the simulated type I error rates for $n=25, p=3,5,10,20, \sigma^{2}=9$, and $\rho=0.6$. From these results it is clear that the CLRT is not a level- $\alpha$ test, even for normally distributed data, when $p$ is close to $n$; and the SIGN test suffers from the same problems as in Figure 1 for non-elliptical data. Consequently, the MCPT is the best choice, with respect to the simulated type I error rates of the three tests for uniform and two-parameter exponential data, even though the MCPT is too liberal in the latter case.

Power

The power of the tests of compound symmetry to detect heteroscedasticity and serial correlation was studied. The MCPT, SIGN test and CLRT were all conducted for various combinations of $n, p$ and distribution; however, because the SIGN test is not a level- $\alpha$ test for uniform and two-parameter exponential data and the CLRT is not a level- $\alpha$ test for double exponential and two-parameter exponential data the power results for these cases are largely excluded in the following discussion, but are presented in Figures 3 and 4 for completeness.

Figure 3 shows the simulated power of the test of compound symmetry versus heteroscedasticity. Specifically, multivariate data were generated from distributions with covariance matrices having diagonal elements given by $1,1+d /(p-1), 1+2 d /(p-1), \ldots, 1+d$ and zero off diagonal elements, where $d$ represents the difference between the first and last (or smallest and largest) diagonal elements. Figure 3 displays the power results for $\mathrm{n}=5,10,25,50$, $p=3, d=4$ and $\rho=0$.

For normally distributed data the power of the CLRT is greater than that for the MCPT and SIGN test in most cases, but the MCPT performs fairly well, achieving a power of 0.983 when $n=50$. The true benefit of the MCPT is observed in the non-normal cases. For uniformly distributed data; the simulated power of the MCPT is greater than or equal to that of the CLRT except for $n=25$ (0.941 for the MCPT and 0.943 for the CLRT). For double exponential data the simulated powers of the MCPT and SIGN test are very close with the MCPT slightly more powerful for small samples $(n=5,10,25)$ and the SIGN test slightly more powerful for large samples $(n=50)$. For two- 


\section{MORRIS, PAYTON \& SANTORICO}

Figure 1: Simulated Type I Error Rates for the Test of Compound Symmetry $\left(p=3, \sigma^{2}=9, \rho=0.6\right)$

a. Normal

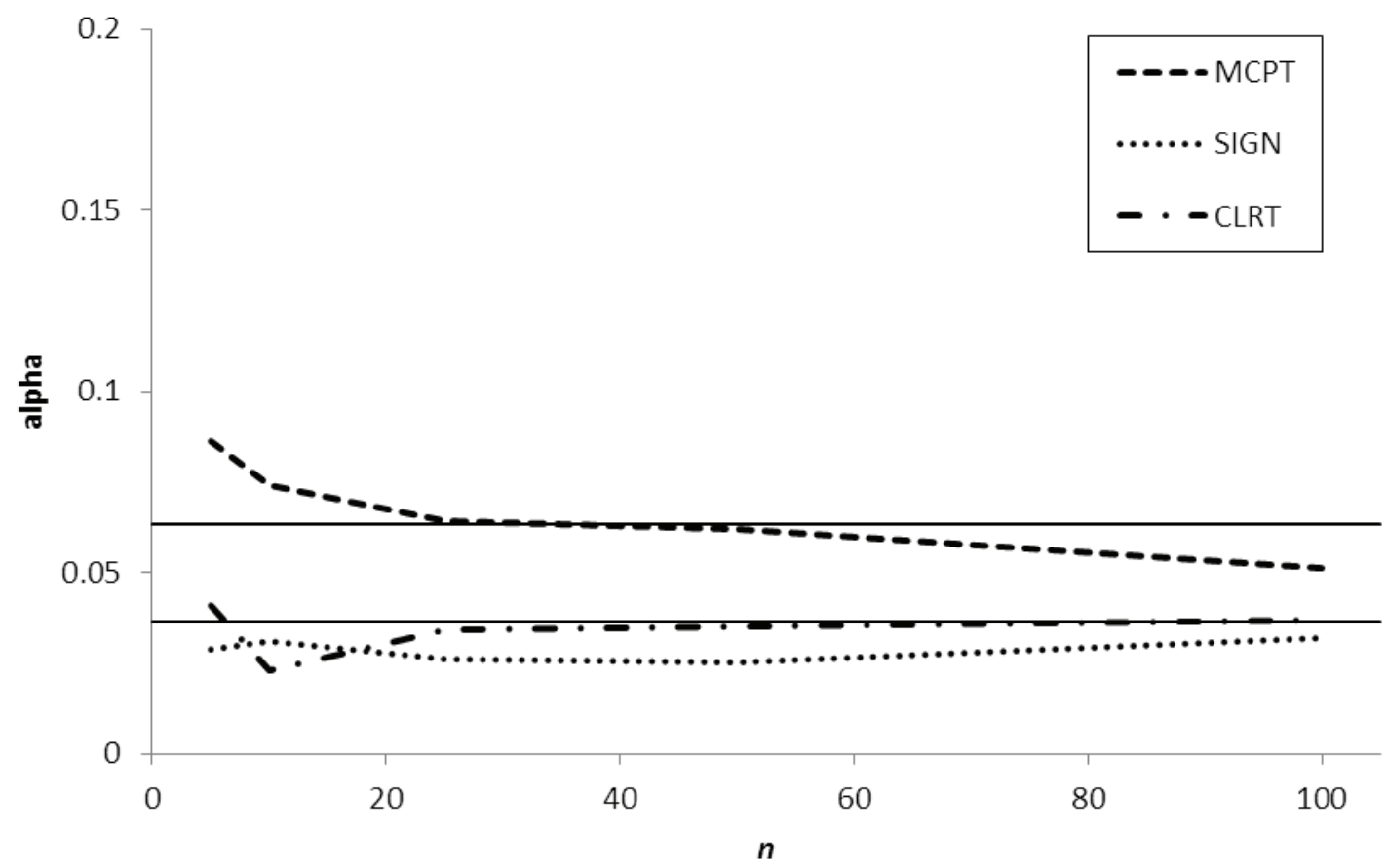

b. Uniform

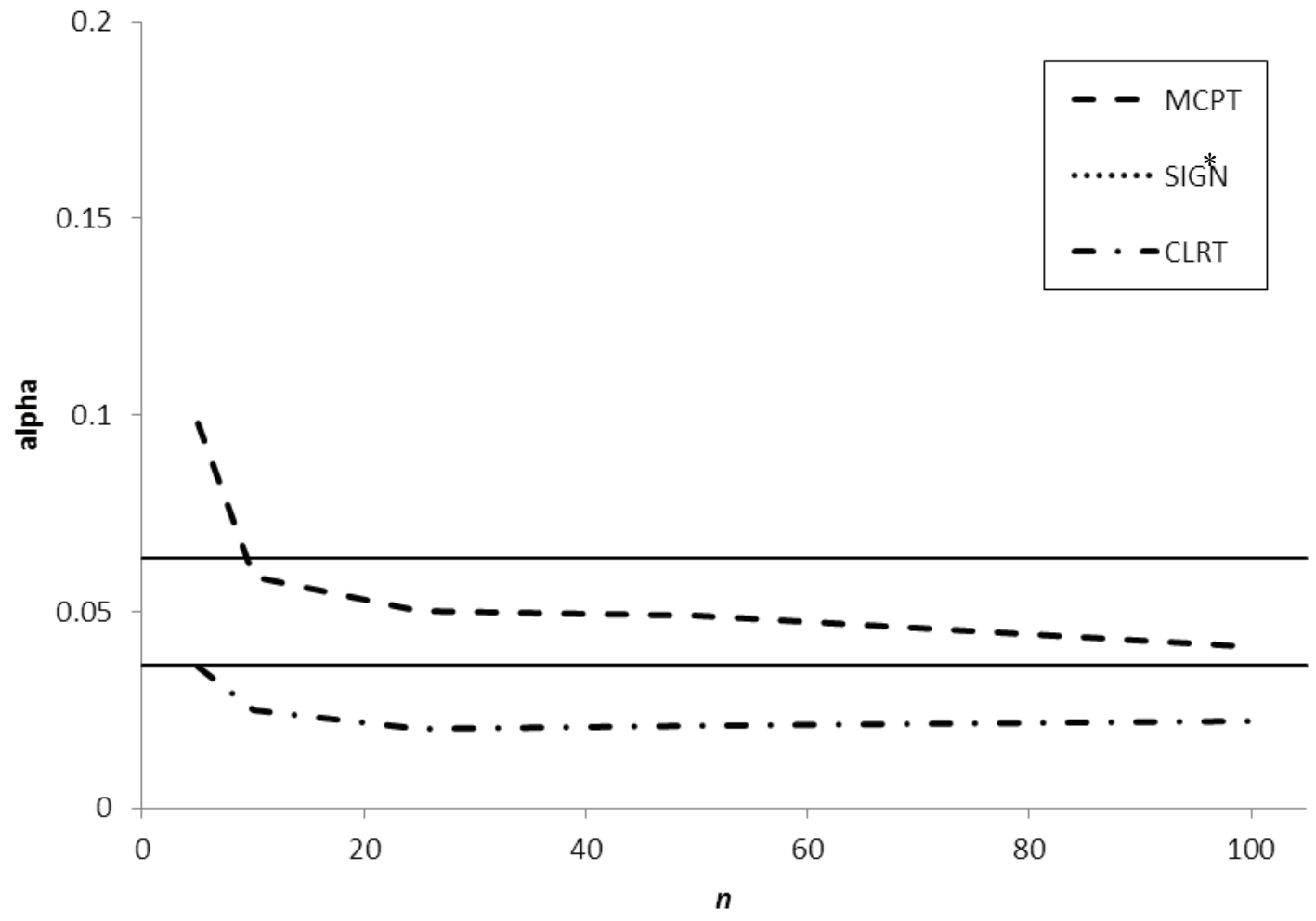

* The type I error rates for this test are greater than 0.2 for all simulated values of $n$.

Note: The horizontal lines correspond to $0.05 \pm 1.96 \sqrt{(0.05)(0.95) / 1000}$ 


\section{A PERMUTATION TEST FOR COMPOUND SYMMETRY}

Figure 1 (continued): Simulated Type I Error Rates for the Test of Compound Symmetry $\left(p=3, \sigma^{2}=9, \rho=0.6\right)$ c. Double Exponential

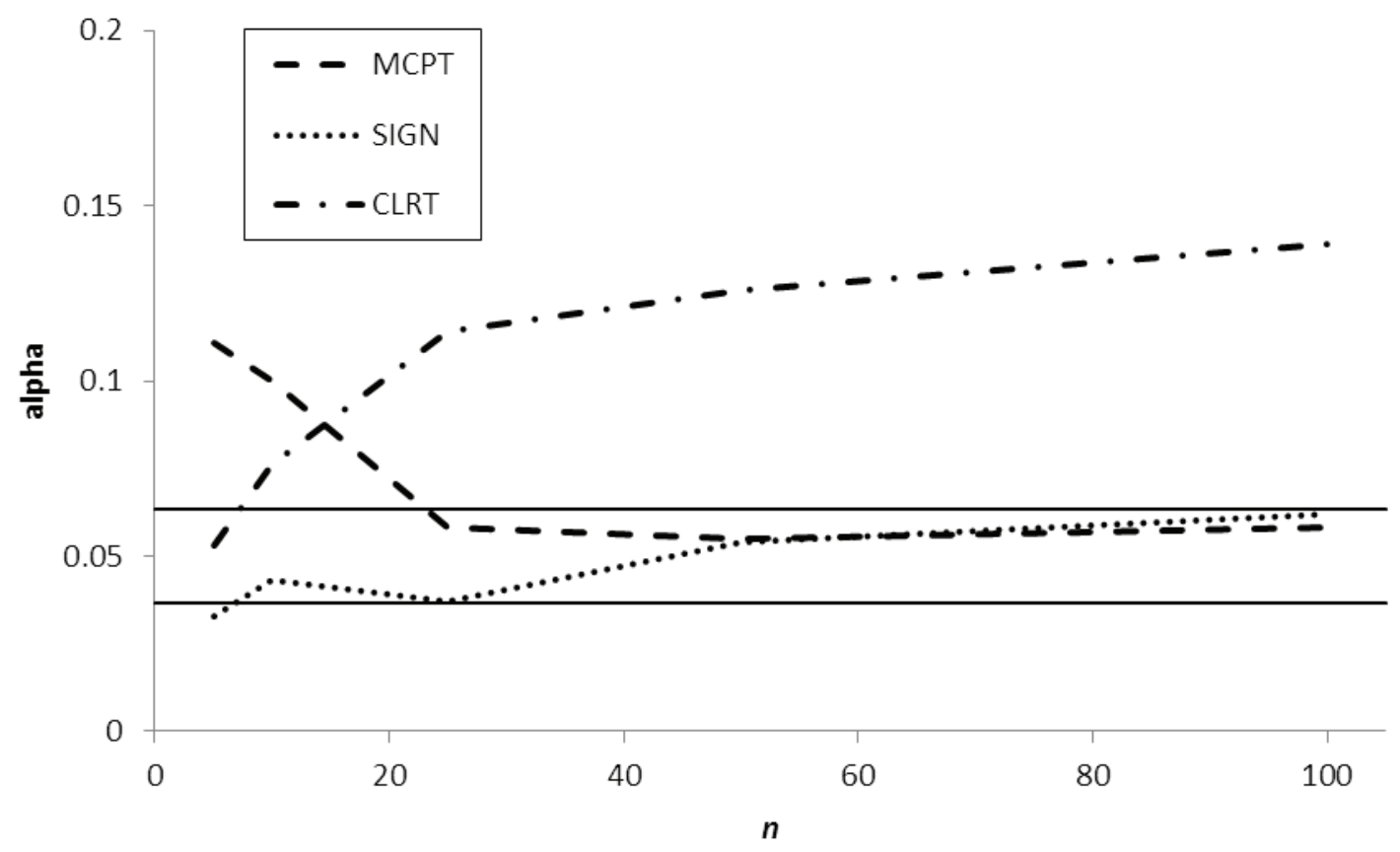

d. Two-Parameter Exponential

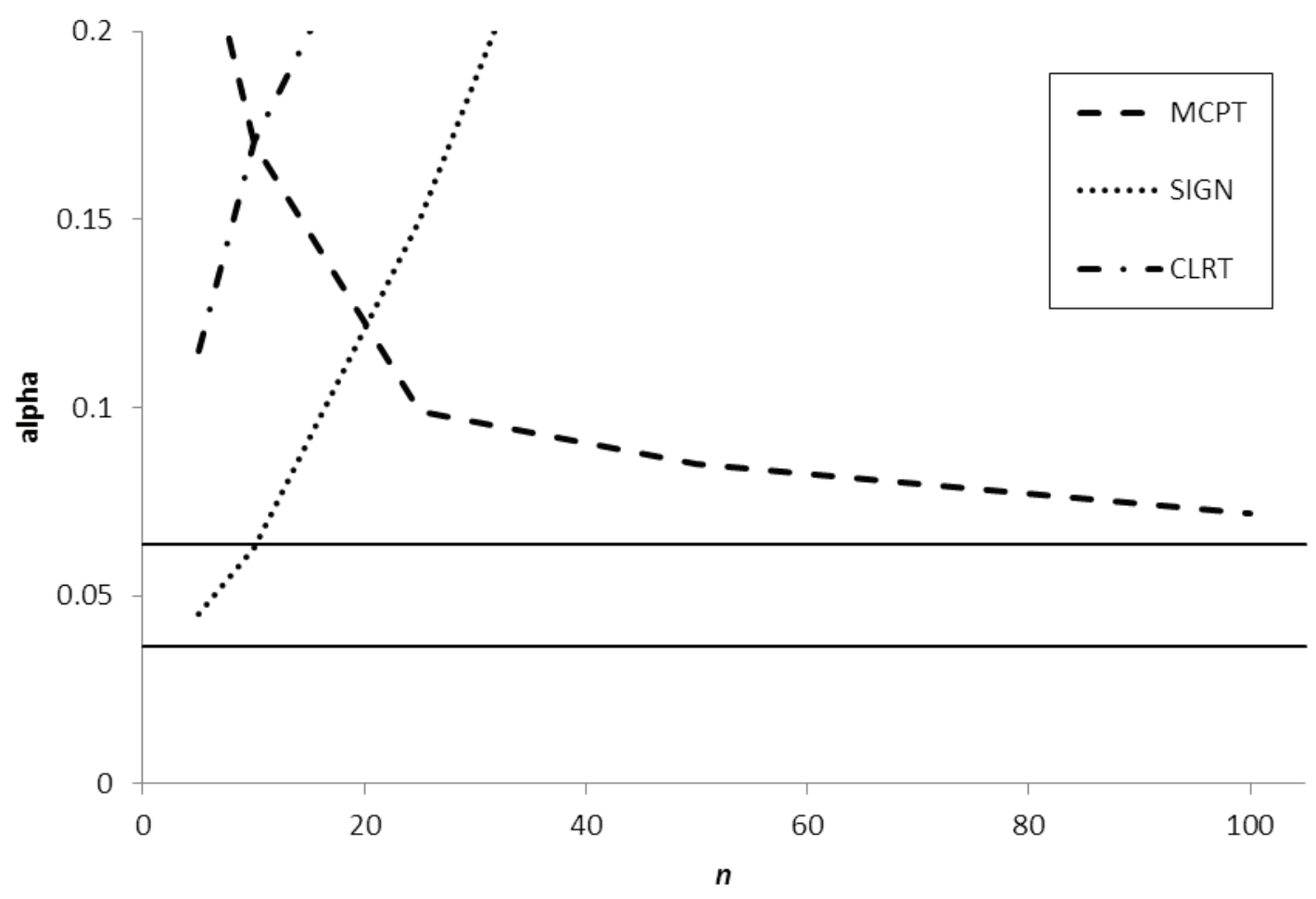

Note: The horizontal lines correspond to $0.05 \pm 1.96 \sqrt{(0.05)(0.95) / 1000}$ 


\section{MORRIS, PAYTON \& SANTORICO}

Figure 2: Simulated Type I Error Rates for the Test of Compound Symmetry $\left(n=25, \sigma^{2}=9, \rho=0.6\right)$

a. Normal

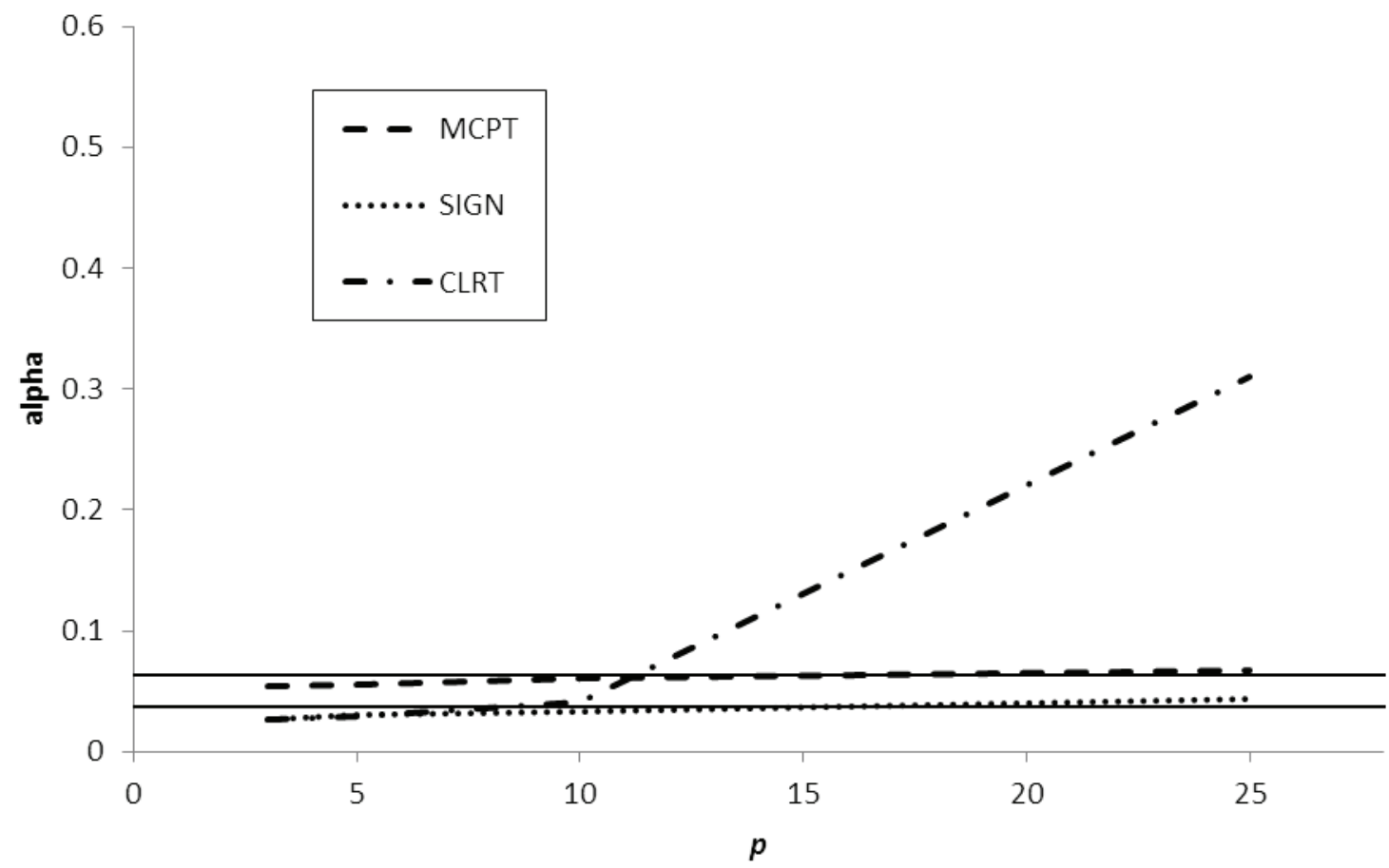

b. Uniform

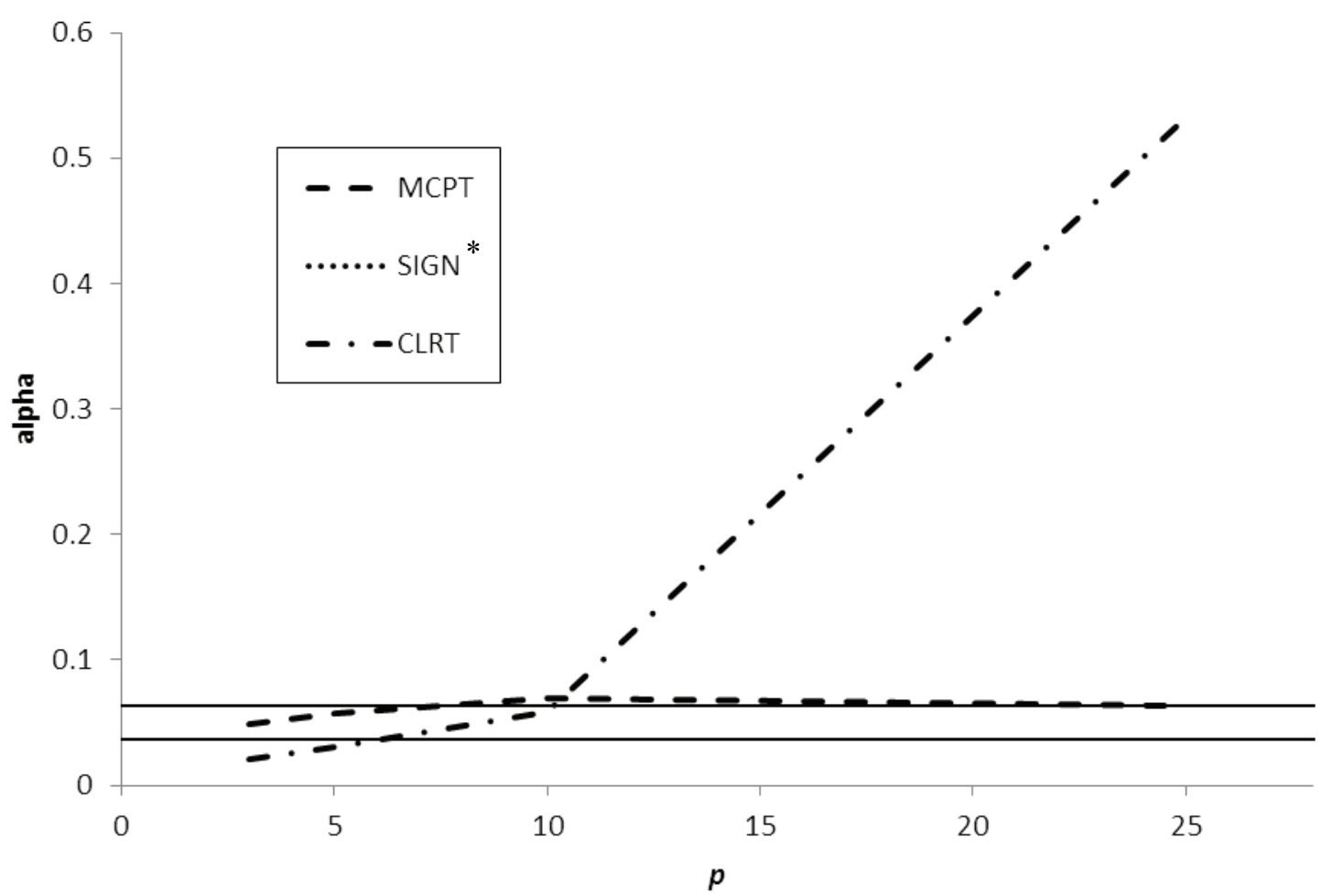

* The type I error rates for this test are greater than 0.2 for all simulated values of $n$.

Note: The horizontal lines correspond to $0.05 \pm 1.96 \sqrt{(0.05)(0.95) / 1000}$ 


\section{A PERMUTATION TEST FOR COMPOUND SYMMETRY}

Figure 2 (continued): Simulated Type I Error Rates for the Test of Compound Symmetry $\left(n=25, \sigma^{2}=9, \rho=0.6\right)$ c. Double Exponential

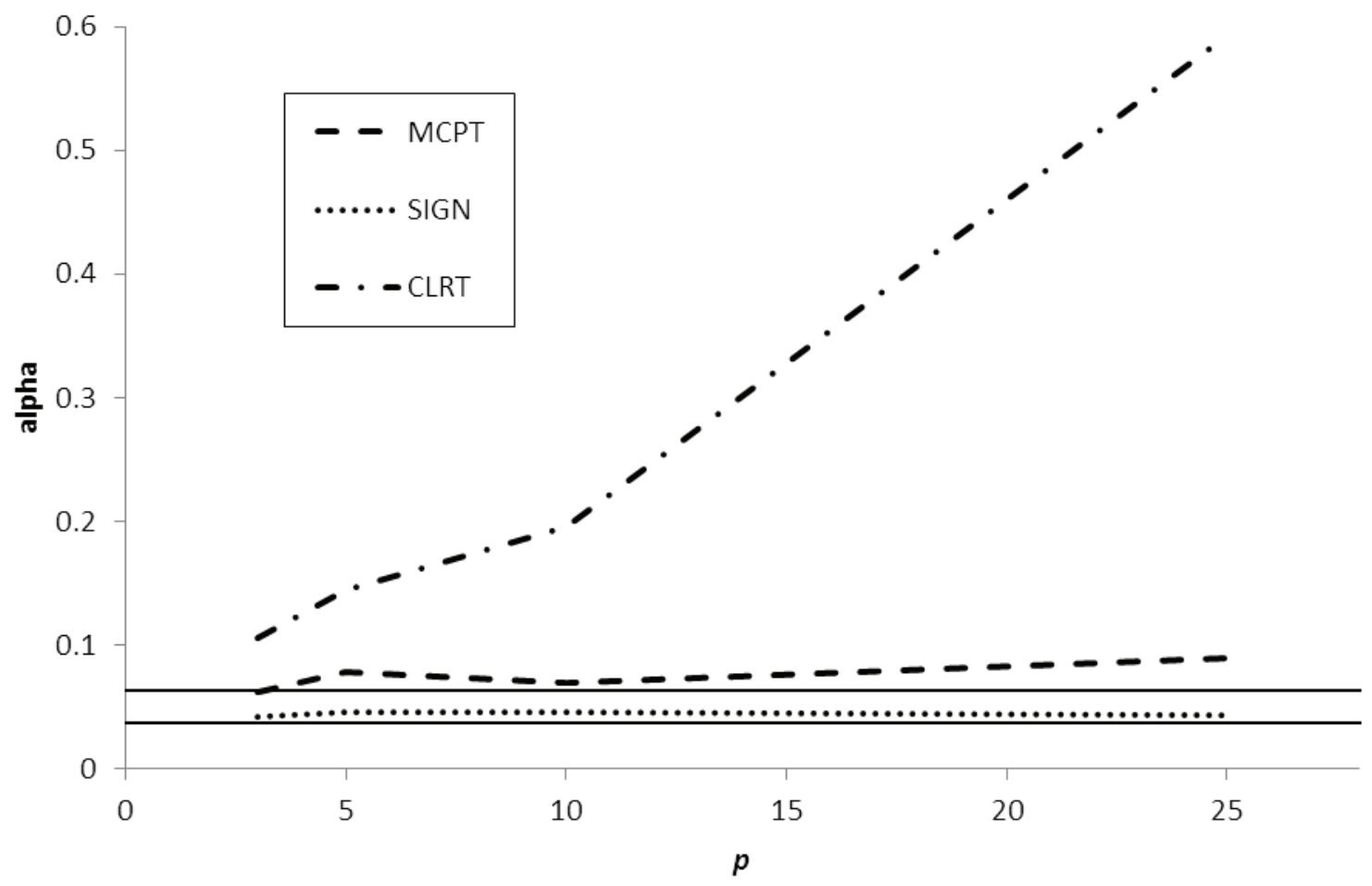

d. Two-Parameter Exponential

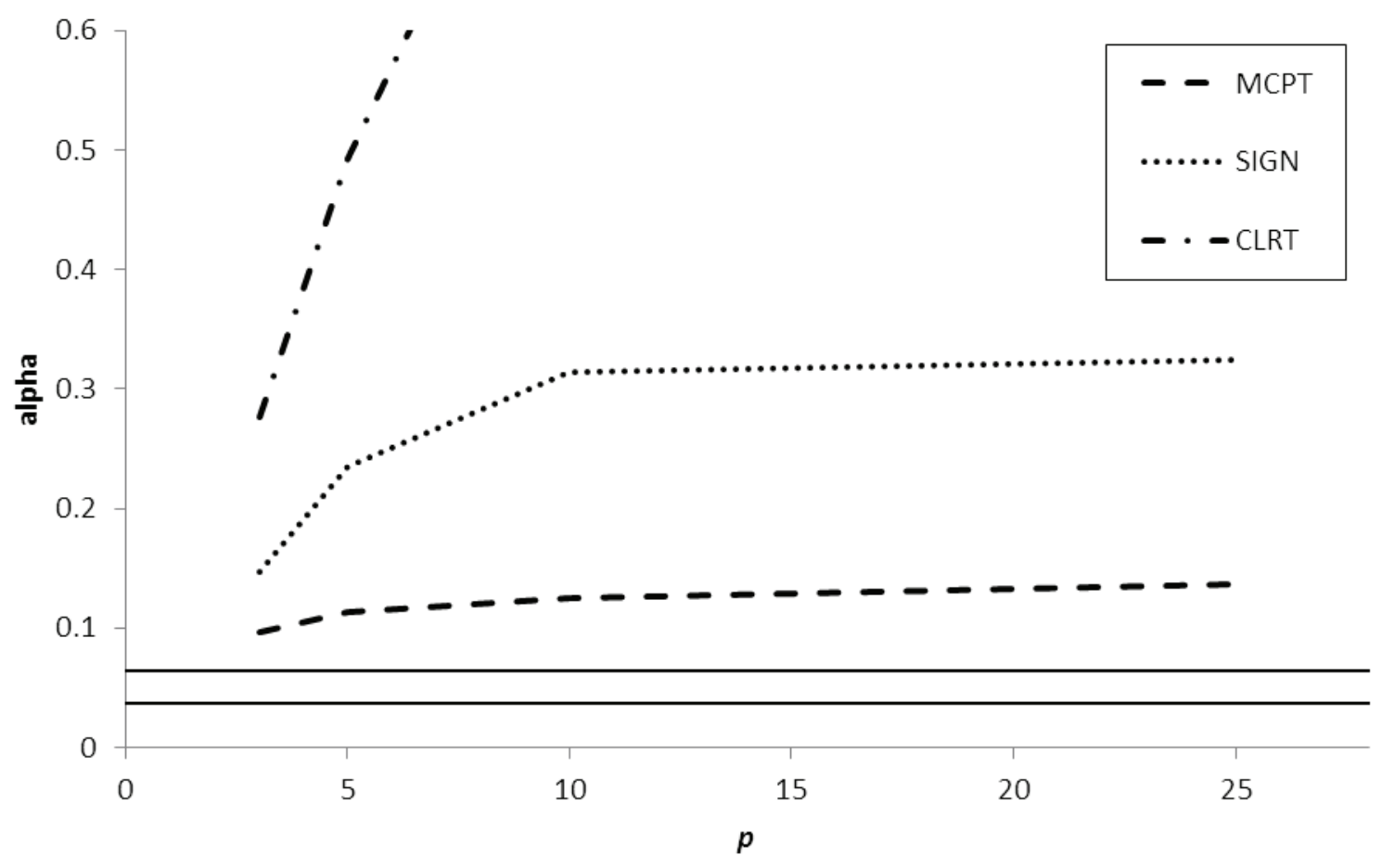

Note: The horizontal lines correspond to $0.05 \pm 1.96 \sqrt{(0.05)(0.95) / 1000}$ 


\section{MORRIS, PAYTON \& SANTORICO}

Figure 3: Simulated Power for the Test of Compound Symmetry vs. Heteroscedasticity $(p=3, \rho=0, d=4)^{*}$ a. Normal

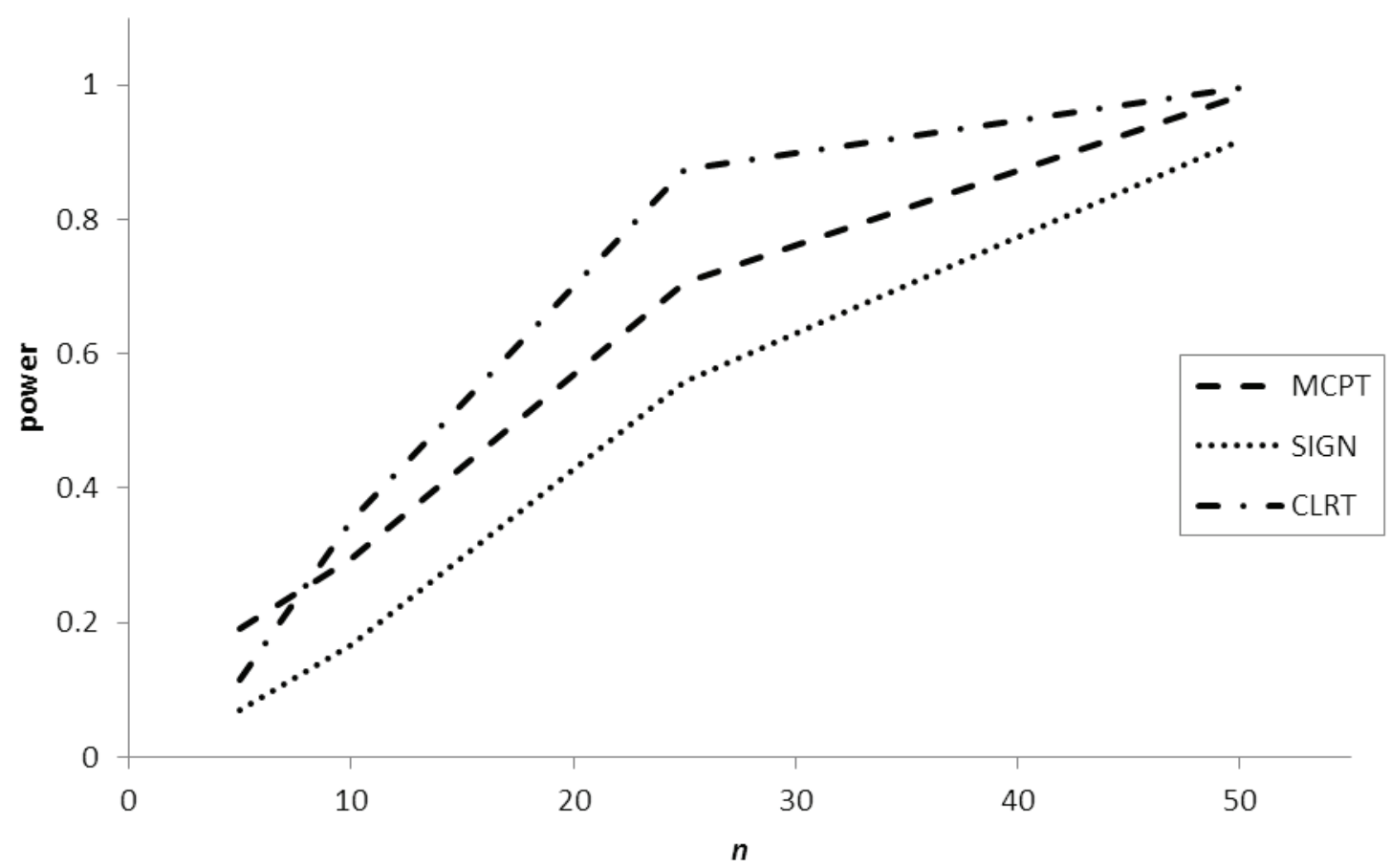

b. Uniform

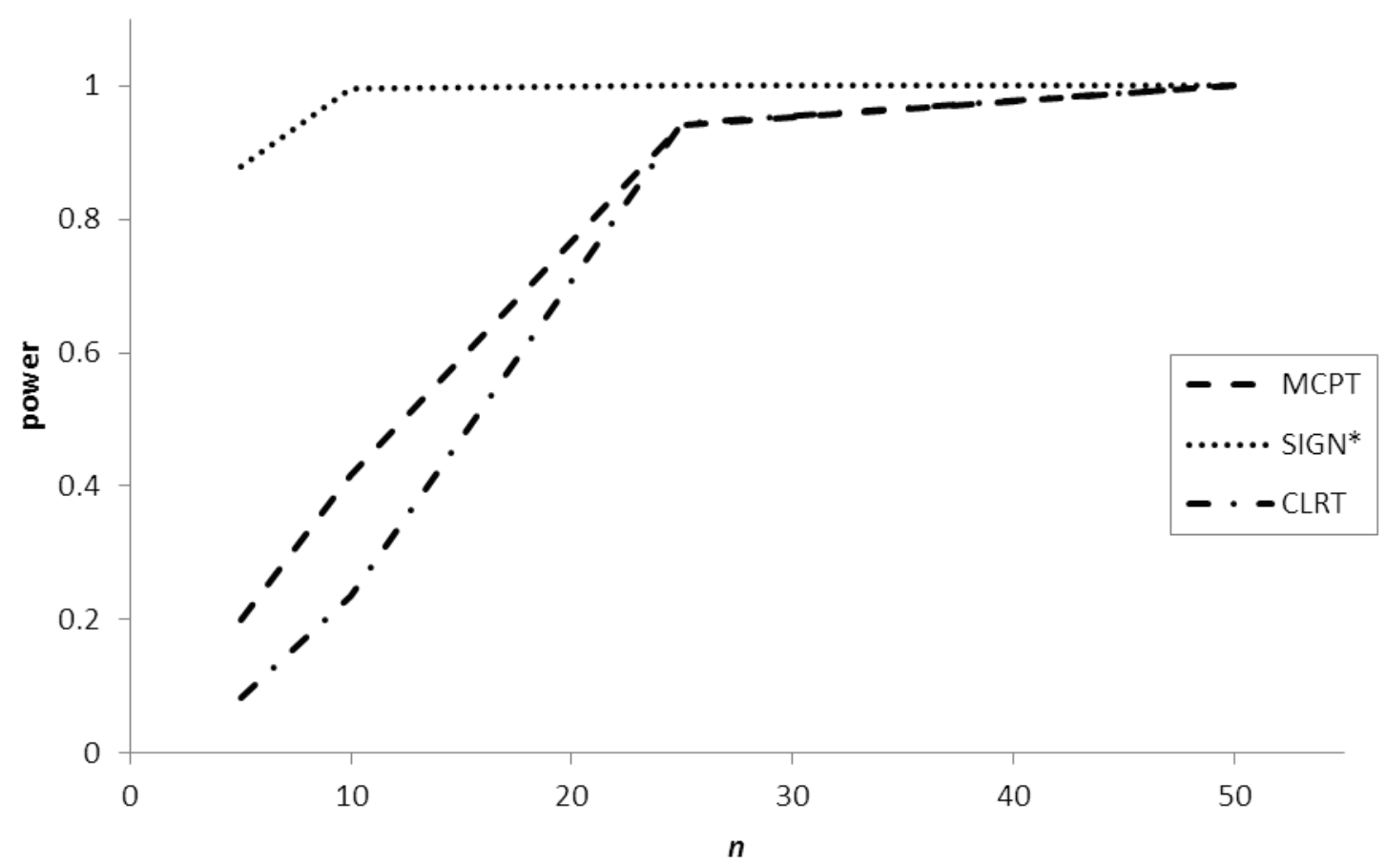

*These are not level- $\alpha$ tests. 


\section{A PERMUTATION TEST FOR COMPOUND SYMMETRY}

Figure 3 (continued): Simulated Power for the Test of Compound Symmetry vs. Heteroscedasticity $(p=3, \rho=0, d=4)^{*}$

c. Double Exponential

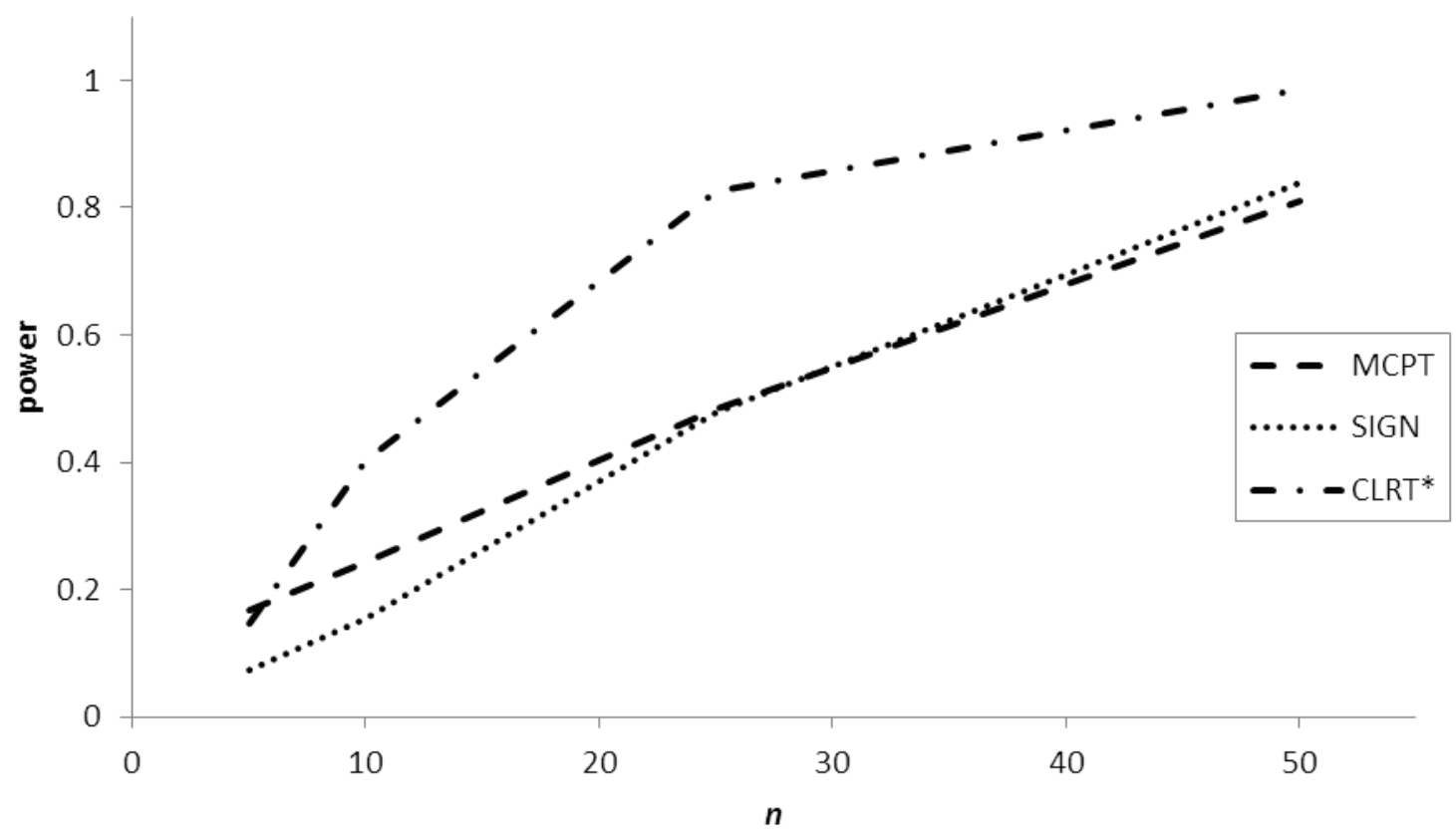

d. Two-Parameter Exponential

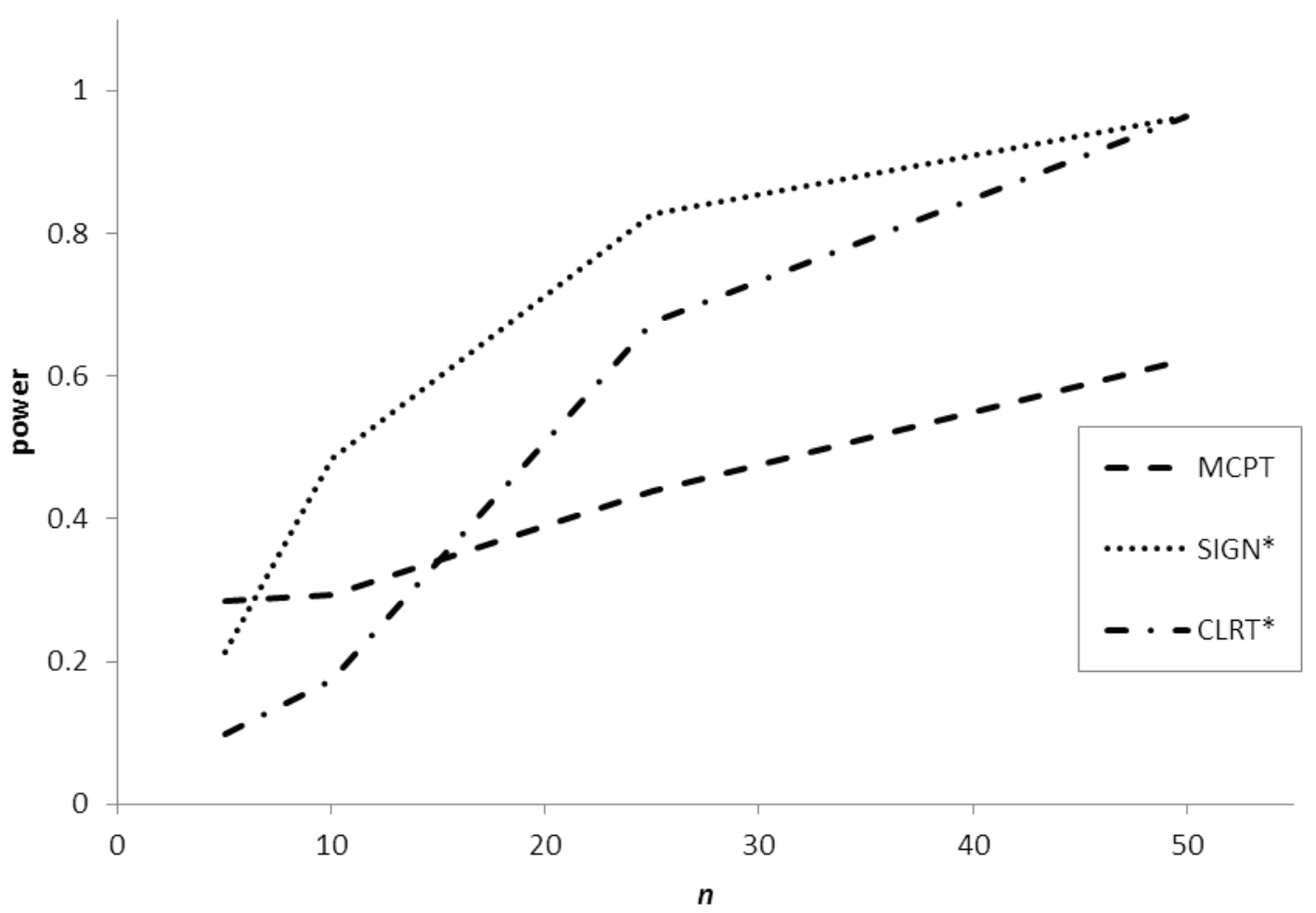

*These are not level- $\alpha$ tests. 
Figure 4: Simulated Power for Test of Compound Symmetry vs. Serial Correlation $\left(p=5, \sigma^{2}=1, \rho=0.6\right)^{*}$ a. Normal

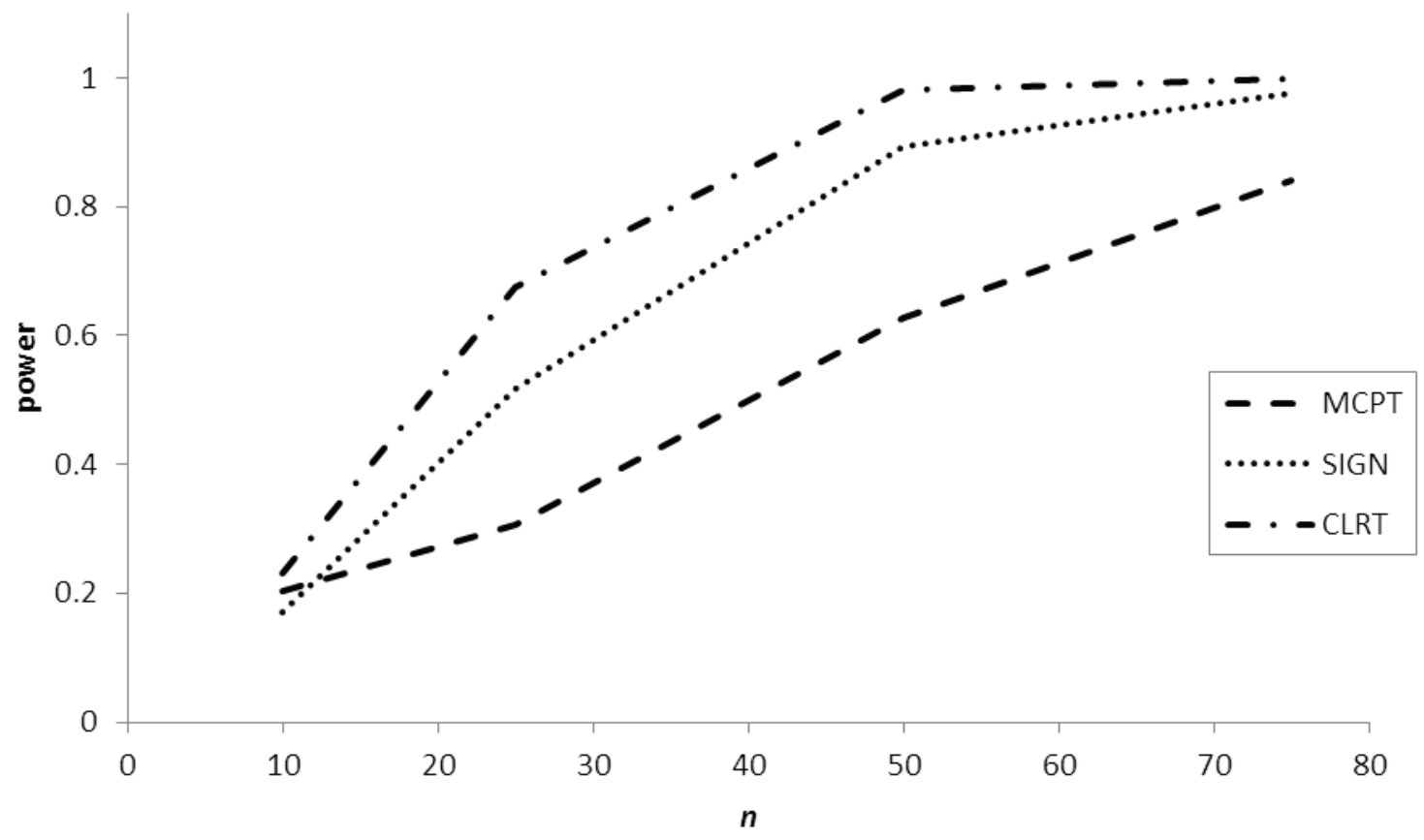

b. Uniform

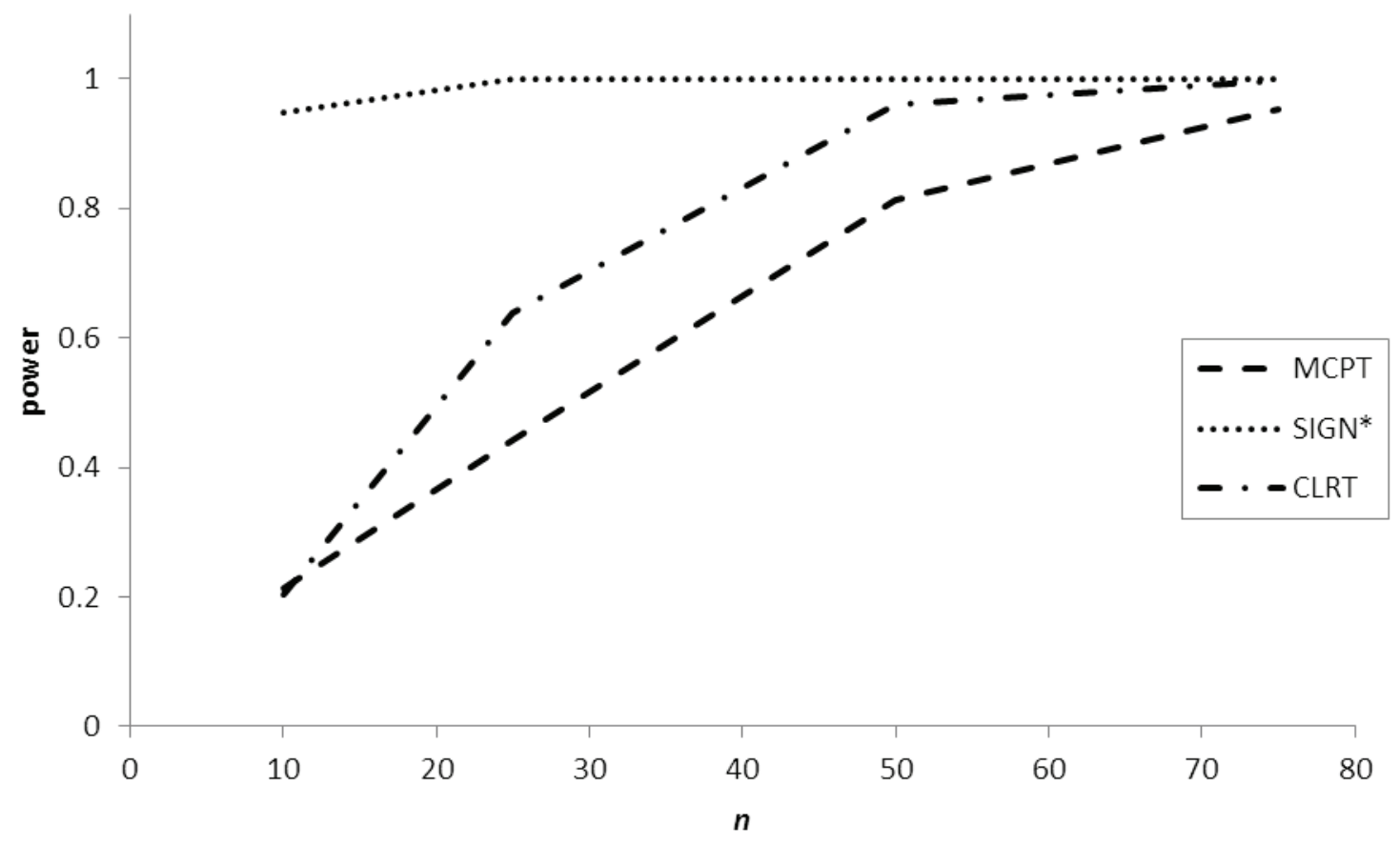

*These are not level- $\alpha$ tests. 


\section{A PERMUTATION TEST FOR COMPOUND SYMMETRY}

Figure 4 (continued): Simulated Power for Test of Compound Symmetry vs. Serial Correlation $\left(p=5, \sigma^{2}=1, \rho=0.6\right)^{*}$ c. Double Exponential

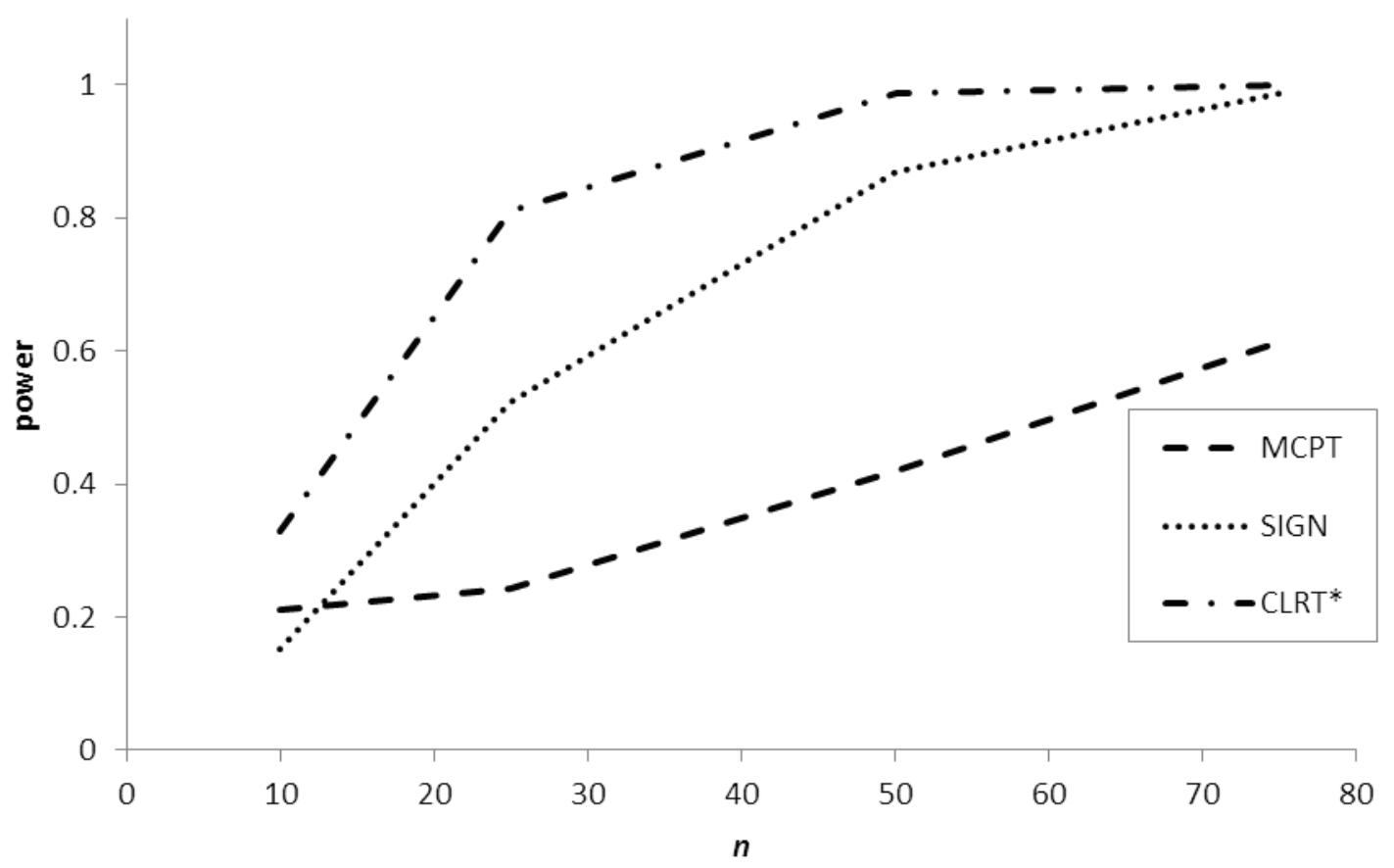

d. Two-Parameter Exponential

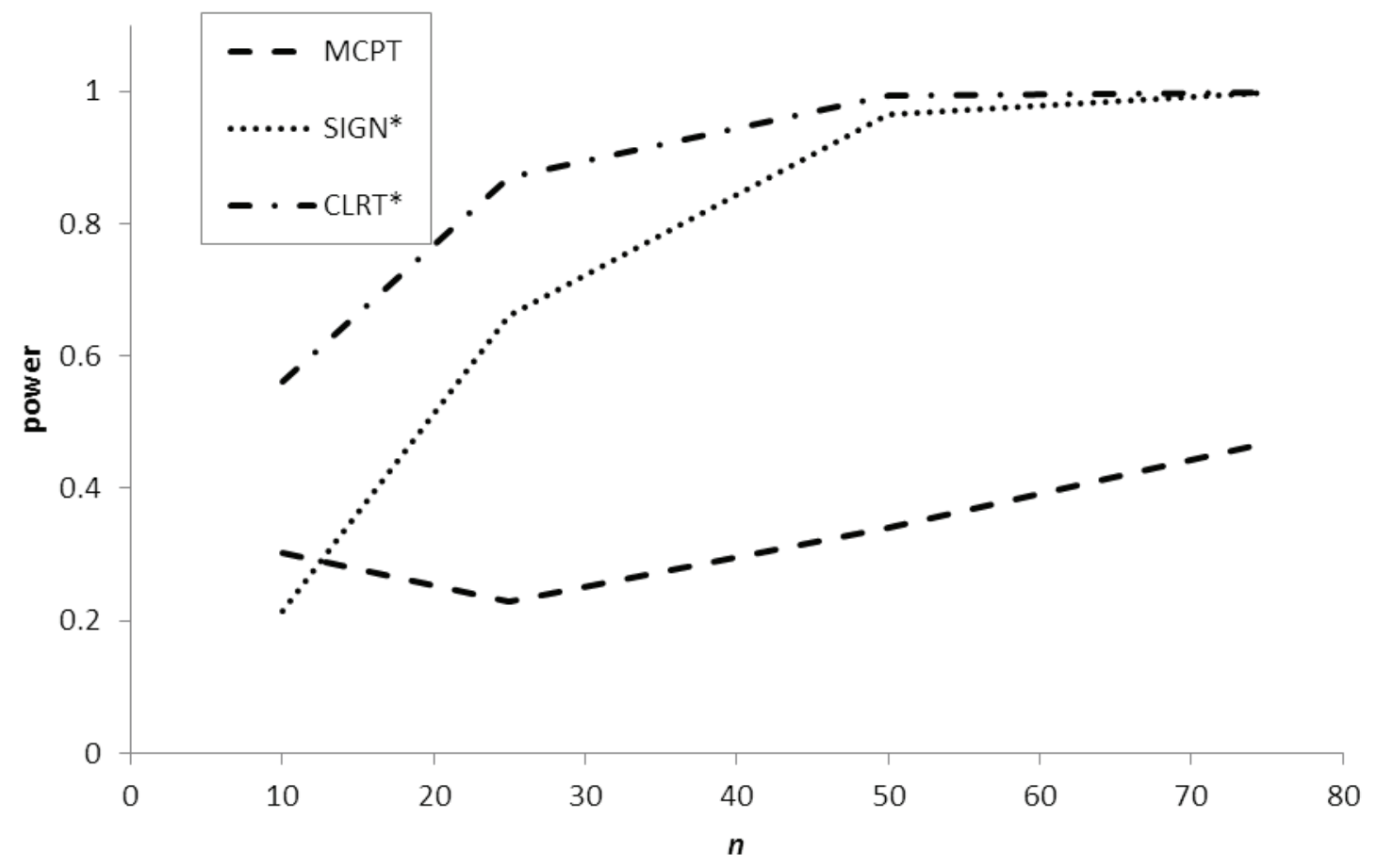

*These are not level- $\alpha$ tests. 


\section{MORRIS, PAYTON \& SANTORICO}

parameter exponential data, even though the MCPT is slightly liberal, it is the best choice of the three tests given that the CLRT and SIGN test have simulated type I error rates that are much too high; however the MCPT in this case is not very powerful, only achieving a simulated power of 0.624 for $n=50$.

Figure 4 displays the simulated power of the test of compound symmetry versus the serial correlation structure given by

$$
\boldsymbol{\Sigma}_{S C}=\frac{\sigma^{2}}{1-\rho^{2}}\left[\begin{array}{ccccc}
1 & \rho & \rho^{2} & \cdots & \rho^{p-1} \\
\rho & 1 & \rho & \cdots & \rho^{p-2} \\
\vdots & \vdots & \vdots & \ddots & \vdots \\
\rho^{p-1} & \rho^{p-2} & \rho^{p-3} & \cdots & 1
\end{array}\right]
$$

where $\sigma^{2} /\left(1-\rho^{2}\right)$ is the common variance of the $p$ variables and $\rho$ is the correlation between successive observations of the variables. Figure 4 displays the power results for $n=10,25,50$, $75, p=5, \sigma^{2}=1$, and $\rho=0.6$.

Figure 4 is very similar to Figure 3 for the CLRT and SIGN test, but the MCPT appears to be less powerful at detecting serial correlation than heteroscedasticity. However, it is difficult to make direct comparisons between these two situations because the degree to which the simulated alternatives depart from compound symmetry cannot be quantified.

\section{Application}

Consider a data set reported in Monks, et al. (2004). In this study, 15 Centre d'Etude du Polymorphisme Humain (CEPH) families were selected and the expression for 23,499 genes was measured in lymphoblastoid cell lines; of these, 762 genes were found to be expressed and heritable. Three of the genes (NM_001081, NM_002125, and V00522) are known to have a linkage to the same location on chromosome 6; consequently, interest lies in determining whether there is a compound symmetry covariance structure with respect to these three genes. Among the 15 families included in the CEPH study there were 47 grandparents. These grandparents were the oldest generation included in the study; therefore, it is assumed that no genetic material is shared among them. Only the 47 grandparents were included in the analysis.

It is common in genetic studies to standardize gene expression data; therefore, the covariance and correlation matrices are equivalent. The sample covariance matrix among these three genes is estimated to be

$$
\hat{\boldsymbol{\Sigma}}=\left[\begin{array}{ccc}
1 & 0.823 & 0.896 \\
0.823 & 1 & 0.824 \\
0.896 & 0.824 & 1
\end{array}\right]
$$

and the hypothesis to be tested is $\mathrm{H}_{0}: \boldsymbol{\Sigma}=\boldsymbol{\Sigma}_{C S}$ vs. $\mathrm{H}_{\mathrm{a}}: \boldsymbol{\Sigma} \neq \boldsymbol{\Sigma}_{C S}$. In all, $(3 !)^{47} \approx 3.74 \times 10^{36}$ permutations of the raw data are possible. Consequently, a random sample of 10,000 permutations was selected for the MCPT. The pvalues for the three tests are 0.9904 for the MCPT, 0.3042 for the CLRT and 0.0664 for the SIGN test. In each case, the null hypothesis would not be rejected at the 0.05 level, but the three p-values are very different. According to the Shapiro-Wilk test of multivariate normality, there is evidence that these data are not from a multivariate normal population $(p=0.00016)$, violating the assumptions of the CLRT. Given that the structure of $\hat{\boldsymbol{\Sigma}}$ does not deviate much from compound symmetry, it may also be speculated that the data may not have a multivariate elliptical distribution which could explain the unusually low p-value for the SIGN test.

\section{Conclusion}

With somewhat recent advances in technology permutation tests are becoming more feasible and - consequently - more common; this article proposed such a test for the compound symmetry covariance structure. Our simulation study indicates that the MCPT is robust to nonnormality (more so when the data are symmetrically distributed), an issue with the CLRT, but is generally not as powerful as the CLRT when the data are normally distributed. The MCPT is also an improvement over the SIGN test in that the MCPT appears to be robust to non-elliptical distributions (again, more so when the data are symmetrically distributed). 


\section{A PERMUTATION TEST FOR COMPOUND SYMMETRY}

One additional - and probably more common situation - that was not considered herein is the case of data sets in which the variables are not all equally distributed. Because the PT requires either independent observations or normally distributed observations with equal covariances for exchangeability, it is suspected that the PT would not perform well in this case, at least for extreme differences in distribution.

This article presented only the PT for the compound symmetry structure. According to Good (2005) this particular test requires multivariate normality and equal covariances for the exchangeability of the data. Evidence presented shows that this test is robust to departures from normality, but the situation of unequal covariances has not been addressed. A data transformation such that a PT for the structure of any covariance matrix can be achieved by applying the PT for compound symmetry to the transformed data is currently under development.

Another issue with the CLRT is that it does not exist for cases in which $p \geq n$. Although the PT exists in these cases, evidence exists to show that it is not a level- $\alpha$ test. Consequently, alternative test statistics are being considered that will alleviate this problem.

\section{Acknowledgements}

This research was supported in part by the Eugene and Doris Miller fellowship of Oklahoma State University and the Office of Research and Grants at the University of Central Oklahoma. The authors would also like to thank Dr. Mauricio Subieta and the High Performance Computer Center at Oklahoma State University for assistance with running the simulations for this study.

\section{References}

Aitkin, M. A. (1969). Some tests for correlation matrices. Biometrika, 56, 443-446.

Aitkin, M. A., Nelson, W. C., \& Reinfurt, K. H. (1968). Tests for correlation matrices. Biometrika, 55, 327-334.
Boik, R. J. (1975). Interactions in the analysis of variance: A procedure for interpretation and a Monte Carlo comparison of univariate and multivariate methods for repeated measures designs. Dissertation Abstracts International, 36, 2908B. (UMI No. 7527837).

Box, G. E. P. (1950). Problems in the analysis of growth and wear curves. Biometrics, 6, 362-389.

Cornell, J. E., Young, D. M., Seaman, S. L., \& Kirk R. E. (1992). Power comparisons of eight tests for sphericity in repeated measures designs. Journal of Educational Statistics, 17, 233-249.

Dwass, M. (1957). Modified randomization tests for nonparametric hypotheses. The Annals of Mathematical Statistics, 28, 181-187.

Falk, M. (1999). A simple approach to the generation of uniformly distributed random variables with prescribed correlations. Communications in Statistics: Simulation and Computation, 28, 785-791.

Fisher, R. A. (1936). The coefficient of racial likeness and the future of craniometry. The Journal of the Anthropological Institute of Great Britain and Ireland, 66, 57-63.

Gao, Y. \& Marden, J. I. (2001). Some rank-based hypothesis tests for covariance structure and conditional independence. Contemporary Mathematics, 287, 97-109.

Good, P. (2005). Permutation, parametric and bootstrap tests of hypotheses $\left(3^{\text {rd }}\right.$ $E d$.). New York, NY: Springer.

Hallin, M., \& Paindaveine, D. (2006). Semiparametrically efficient rank-based inference for shape I. Optimal rank-based tests for sphericity. The annals of statistics, 34, 27072756.

Huynh, H., \& Mandeville, G.K. (1979). Validity conditions in repeated measures designs. Psychological Bulletin, 86, 964-973.

Jöckel, K. H. (1986). Finite sample properties and asymptotic efficiency of Monte Carlo tests. The Annals of Statistics, 14, 336347.

Keselman, H. J., Rogan, J. C., Mendoza, J. L., \& Breen, L. J. (1980). Testing the validity conditions of repeated measures $F$ tests. Psychological Bulletin, 87, 479-481. 


\section{MORRIS, PAYTON \& SANTORICO}

Lee, J. C., Krishnaiah, P. R., \& Chang, T. C. (1976). On the distribution of the likelihood ratio test statistic for compound symmetry. South African Statistical Journal, 10, 49-62.

Manly, B. F. J. (1997). Randomization and Monte Carlo methods in biology ( $2^{\text {nd }}$ ed.). London: Chapman \& Hall.

Marden, J. I. (1999). Multivariate rank tests. In S. Ghosh (Ed.), Multivariate analysis, design of experiments, and survey sampling, 401-432. New York, NY: Marcel Dekker.

Marden, J., \& Gao, Y. (2002). Rankbased procedures for structural hypotheses on covariance matrices. Sankhyā: the Indian Journal of Statistics, 64, 653-677.

Mauchly, J. W. (1940). Significance test for sphericity of a normal $n$-variate distribution. The Annals of Mathematical Statistics, 11, 204209.

Monks, S. A., et al. (2004). Genetic inheritance of gene expression in human cell lines. American Journal of Human Genetics, 75, 1094-1105.

R Development Core Team (2009). $R: A$ language and environment for statistical computing (Version 2.10.1). Available from http://www.R-project.org.
Sirkiä, S., Nordhausen, K., \& Oja, H. (2009). SpatialNP: Multivariate nonparametric methods based on spatial signs and ranks (Version 1.0-1). Available from http://CRAN.Rproject.org/package $=$ SpatialNP.

Sirkiä, S., Taskinen, S., Oja, H., \& Tyler, D. E. (2009). Tests and estimates of shape based on spatial sings and ranks. Journal of Nonparametric Statistics, 21, 155-176.

Vale, C. D., \& Maurelli, V. A. (1983). Simulating multivariate nonnormal distributions. Psychometrika, 48, 465-471.

Venables, W. N., \& Ripley, B. D. (2002). Modern applied statistics with $S\left(2^{\text {nd }}\right.$ $E d$.). New York: NY: Springer.

Votaw, D. F. (1948). Testing compound symmetry in a normal multivariate distribution. The Annals of Mathematical Statistics, 19, 447473.

Wilks, S. S. (1946). Sample criteria for testing equality of means, equality of variances, and equality of covariances in a normal multivariate distribution. The Annals of Mathematical Statistics, 17, 257-281. 